\title{
DarkSide-20k and the Future Liquid Argon Dark Matter Program
}

\author{
Bianca Bottino $^{a, b, 1, *}$ \\ a Princeton University \\ Jadwin Hall, Washington Road, Princeton, New Jersey, USA \\ ${ }^{b}$ Istituto Nazionale di Fisica Nucleare, Sez. di Genova \\ Via Dodecaneso 33, Genoa, Italy \\ E-mail: bianca.bottino@ge.infn.it
}

DarkSide ran since mid 2015 a 50-kg-active-mass dual-phase Liquid Argon Time Projection Chamber (TPC), filled with low-radioactivity argon from an underground source and produced world class results for both the low mass $\left(\mathrm{M}_{W I M P}\right.$ below $\left.20 \mathrm{GeV} / \mathrm{c}^{2}\right)$ and high mass $\left(\mathrm{M}_{W I M P}\right.$ above $100 \mathrm{GeV} / \mathrm{c}^{2}$ ) direct detection search for dark matter. The next stage of the DarkSide program will be a new generation experiment, called DarkSide-20k, involving a global collaboration formed by members of the main current argon-based experiments. DarkSide-20k, is designed as a 20tonne fiducial mass dual-phase Liquid Argon TPC with SiPM-based cryogenic photosensors, and is expected to be free of any instrumental background for an exposure of $>100 \mathrm{t} \cdot \mathrm{yr}$. Like its predecessor, DarkSide-20k will be housed at the INFN Gran Sasso underground laboratory (LNGS), and it is expected to attain a WIMP-nucleon cross section $90 \%$ C.L. exclusion sensitivity of $7.4 \times 10^{-48} \mathrm{~cm}^{2}$ for a WIMP mass of $1 \mathrm{TeV} / \mathrm{c}^{2}$ in a $200 \mathrm{t} \cdot \mathrm{yr}$ run. DarkSide-20k will be installed inside a membrane cryostat containing more than $700 \mathrm{t}$ of liquid argon, and be surrounded by an active neutron veto based on a Gd-loaded acrylic shell. The article will give the latest updates of the ongoing R\&D and prototype tests validating the initial design. A subsequent objective, towards the end of the next decade, will be the construction of the ultimate detector with a $300 \mathrm{t}$ fiducial mass, Argo, to push the sensitivity to the neutrino floor region for high-mass WIMPs.

\footnotetext{
*** The European Physical Society Conference on High Energy Physics (EPS-HEP2021), ***

*** 26-30 July $2021 * * *$

*** Online conference, jointly organized by Universität Hamburg and the research center DESY ***
}

\footnotetext{
${ }^{1}$ on behalf of the DarkSide Collaboration

* Speaker
} 


\section{The use of liquid argon for dark matter searches}

There is strong evidence from astronomical and cosmological observations for the existence of dark matter in our Universe. Weakly Interacting Massive Particles (WIMPs) are a well-motivated dark matter candidate that may have been produced in the early Universe, but are so weakly interacting that they have yet to be observed in a terrestrial experiment.

Earth-based direct detection experiments aim to detect galactic dark matter particles via their interaction with ordinary matter in an instrumented target. Noble liquid detectors, in the dualphase Time Projection Chamber (TPC) layout, are particularly suitable for these searches, thanks to scalability, intrinsic radio-purity and good ionization and scintillation properties of the target. The TPC consists of an active volume filled with liquid, which is immersed in a uniform electric field, to drift ionization electrons towards a gaseous region on top of the detector, where charges are accelerated to stimulate light production by electro-luminescence. Photo-detectors collect both the prompt scintillation light produced in liquid (S1), and the delayed electro-luminescence light (S2) generated in gas, allowing for energy measurement and 3D vertex reconstruction.

In addition to this, liquid argon (LAr) has excellent event discrimination capabilities. Scintillation light initiated by particles recoiling from atomic electrons, that is the primary source of background in a WIMP direct detection experiment, has a time constant of approximately a microsecond. This is in stark contrast to the nanosecond time constant of scintillation light emitted during an expected WIMP-nuclear recoil event. The DEAP-3600 experiment has exploited this effect via pulse shape discrimination (PSD) to achieve electron recoil background rejection of $2.4 \times 10^{8}[1,2]$. DarkSide50 , whose experimental layout is described extensively in[3], has presented results from a 532,4 live-days exposure, and observed no background events over a run period in excess of two years [7]. In addition to sensitivity to WIMPs with masses above $100 \mathrm{GeV} / \mathrm{c}^{2}$, the two-phase DarkSide-50 detector has extended its reach to WIMP masses below $20 \mathrm{GeV} / \mathrm{c}^{2}$ by detecting single ionizaton electrons extracted from the liquid argon volume $[8,10]$. These results were possible as argon extracted from deep underground sources (UAr) was used as the active medium, to avoid the $1 \mathrm{~Bq} / \mathrm{kg}{ }^{39} \mathrm{Ar}$ activity in atmospheric argon produced by cosmic-ray activation. DarkSide-50 has demonstrated that using underground argon the rate of ${ }^{39} \mathrm{Ar}$ events could be reduced by a factor of 1400 [4].

\section{Future liquid argon dark matter program}

Given the discovery potential of an argon-based detector, scientists from all of the major experiments currently using LAr to search for dark matter, including ArDM, DarkSide-50, DEAP3600, and MiniCLEAN, have joined to form the Global Argon Dark Matter Collaboration (GADMC) with the goal of building a series of future experiments that maximally exploit the advantages of LAr as a detector target. The immediate objective of the GADMC is the construction of the DarkSide$20 \mathrm{k}$ two-phase LAr detector filled with $50 \mathrm{t}$ of radio-pure argon extracted from underground sources, which will operate in Hall-C of the INFN Gran Sasso National Laboratory (LNGS). The DarkSide20k detector will have ultra-low backgrounds and the ability to measure its backgrounds in situ, resulting in an expected $90 \%$ C.L. sensitivity to WIMP-nucleon cross sections of $7.4 \times 10^{-48} \mathrm{~cm}^{2}$ for a WIMP mass of $1 \mathrm{TeV} / \mathrm{c}^{2}$ with an exposure of $200 \mathrm{t} \cdot \mathrm{yr}$ run. To achive this objective DarkSide-20k 


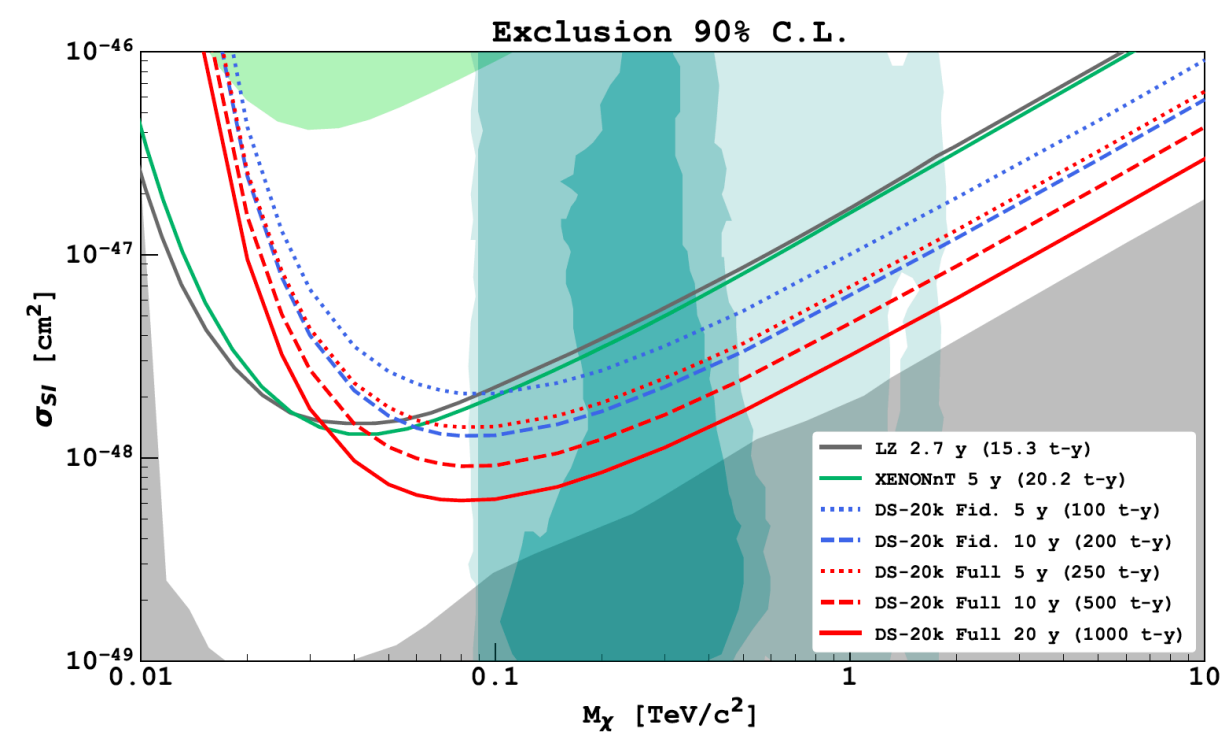

Figure 1: The sensitivity of DarkSide-20k to spin-independent WIMPs for different exposures shown compared to the nominal sensitivity of currently-funded experiments, that are expected to lead the field for high-mass WIMP searches in the next years [5, 6]. The region excluded by XENON1T is shown in shaded green, the "neutrino floor" in shaded gray, and the turquoise filled contours represent the 1- $\sigma, 2-\sigma$, and 3- $\sigma$ favored regions from the pMSSM11 model constrained by astrophysical measurements and the $\sim 36 \mathrm{fb}^{-1}$ LHC data at $13 \mathrm{TeV}[9]$.

is designed to operate almost background free, meaning that all sources of instrumental background are reduced to $<0.1$ events over a $200 \mathrm{t}$ yr exposure (see Fig. 1). Constructing the detector will require a very large amount of UAr to be procured in a timely fashion. This will be accomplished by Urania, an argon extraction and purification plant capable of extracting $330 \mathrm{~kg} / \mathrm{d}$ of UAr. Further purification of UAr will be carried out in the Aria plant, with a $350 \mathrm{~m}$ tall cryogenic distillation column that is currently in the installation phase in a mine shaft at Carbosulcis, in Sardinia, Italy. A $26 \mathrm{~m}$ pilot plant, which consists of a bottom reboiler, a top condenser, and a single central module, has been installed above-ground site and was successfully tested [12].

The ultimate objective of the GADMC is the construction of the Argo detector, which will have a $300 \mathrm{t}$ fiducial mass and will push experimental sensitivity to the point at which the coherent scattering of atmospheric neutrinos becomes a limiting background. The excellent electron recoil (ER) rejection possible in argon will eliminate backgrounds from solar neutrinos, which will extend the sensitivity of Argo beyond that of technologies with more limited ER discrimination.

\section{DarkSide-20k detector}

The DarkSide-20k detector consists of two nested detectors housed within a ProtoDUNE-style membrane cryostat [11], as shown in the sketch in Figure 2. The inner detector is a dual-phase argon TPC contained within an octagonal vessel made from ultra-pure acrylic (PMMA) and filled with underground argon. The central active volume of the TPC is defined by eight vertical reflector panels and the top and bottom windows of the acrylic vessel. All the TPC surfaces in contact with the active 


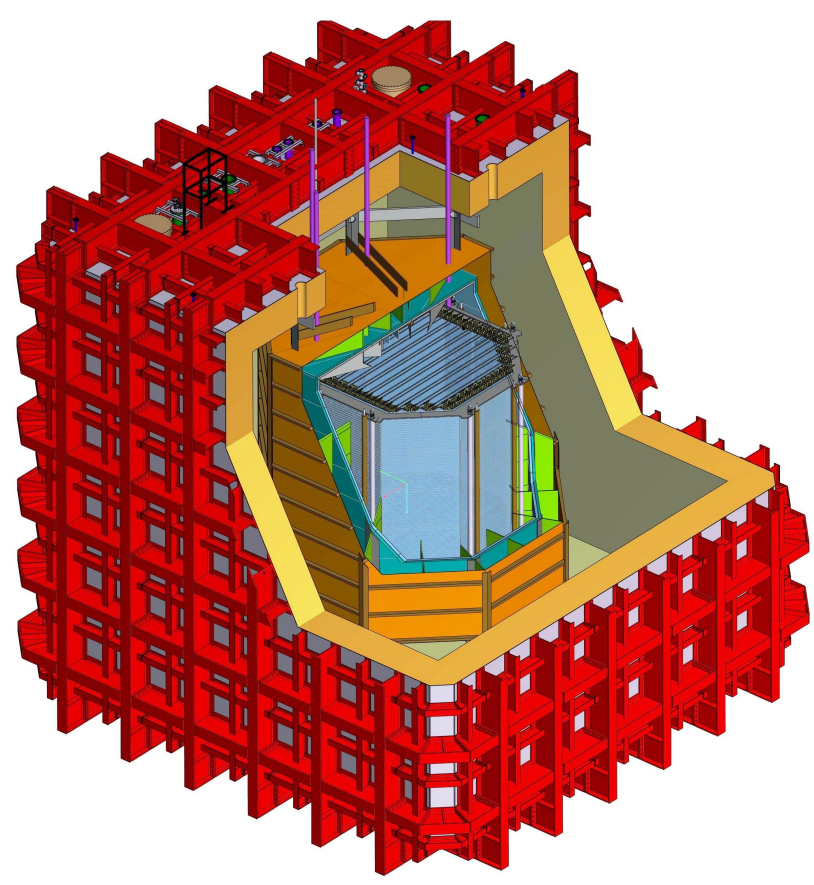

Figure 2: DarkSide-20k detector. The sealed acrylic (PMMA) TPC (light blue) is surrounded by the veto detector, which consists of a Gd-loaded acrylic shell (green) sandwiched between two atmospheric argon (AAr) active layers, and contained in a ProtoDUNE-like cryostat (red). The outer active argon layer is separated from the rest of the AAr by a copper vessel (orange). The TPC is holded by a support structure (purple), hanging from the top of the cryostat.

argon volume will be coated with a tetraphenyl-butadiene wavelength shifter (TPB) to convert LAr scintillation light to a wavelength detectable at high efficiency by silicon photomultipliers (SiPMs). 8280 SiPM-based Photodetector Modules (PDMs) will view the argon volume through the top and bottom windows of the acrylic vessel. The height of the TPC is $350 \mathrm{~cm}$. The total mass of LAr in the active volume is $49.7 \mathrm{t}$. Around the TPC there will be the neutron veto detector, discussed in the following section. Both detectors will operate within a membrane cryostat filled with liquefied atmospheric argon, a technology initially developed at CERN for ProtoDUNE.

The PDM for the TPC consists of a large tile of SiPMs covering a total area of $50 \times 50 \mathrm{~mm}^{2}$ operating as a single detector. Besides the tile, each module will also contain a cryogenic preamplifier board that will amplify and shape the signal in the immediate proximity of the sensor. In order to maximize the amplification factor while preserving a stable signal bandwidth and SNR, the Collaboration has developed and optimized a transimpedance amplifier with excellent performance at $87 \mathrm{~K}$.

\section{A zoom on the Veto detector}

The Veto detector is composed of three separate volumes: a passive octagonal shell made of gadolinium-loaded PMMA, mounted around the TPC and providing to the TPC a $4 \pi$ coverage; a 40 $\mathrm{cm}$ thick inner volume of active liquid atmospheric argon (AAr) and sandwiched between the TPC vessel and the Gd-loaded PMMA; a $40 \mathrm{~cm}$ thick outer active volume of AAr contained between 
the Gd-loaded PMMA and the outer copper Faraday cage. The Faraday cage contains the veto and TPC detectors, and insulates them optically and electrically from the remaining, outermost AAr volume contained within the cryostat. From Monte Carlo simulations the Gd-loaded acrylic thickness has to be of $10 \mathrm{~cm}$, with a Gd concentration in mass between $1 \%$ and $2 \%$. The acrylic moderates neutrons produced in the detector materials and the inclusion of the Gd enhances the neutron capture probability. The capture of the neutron on a $\mathrm{Gd}$ nucleus results in the emission of multiple $\gamma$-rays. These $\gamma$-rays interact in the AAr layers and cause the emission of scintillation light that is detected by photodetectors, thereby providing an efficient veto of radiogenic neutrons that could result in a nuclear recoil in the TPC. In order to optimize the light readout and minimize the overall ${ }^{39} \mathrm{Ar}$ rate in each detector element, the argon buffers are segmented into eight parts by subdivision plates coated with a light reflector and a wavelength shifter.

In order to meet the radiopurity requirements of the experiment, an ultra-pure type of gadolinium oxide was selected to be mixed with the acrylic. Since gadolinium oxide is not miscible with liquid MMA (the liquid monomer of the acrylic), it was necessary to develop a proper mixing procedure. The strategy adopted to obtain a uniform distribution of $\mathrm{Gd}$ was to use gadolinium oxide in the form of nano-grains, performing a functionalization of the particles before the polymerization process. Lab-scale test are very promising and the next phase will be to produce samples in an industrial line.

The Veto PDMs will be mounted on the two sides of the Gd-loaded shell, such that they are facing both the inner argon buffer and the outer one. About 3000 SiPM tiles in total are foreseen, 2000 of them facing the inner buffer and 1000 facing the outer buffer. The basic optical detector element is the same SiPM tile used in the TPC, but with a different front end board (FEB) coupled to it, that is optimized for the geometry and the electronics of the veto detector. The baseline option is the use of a front-end board realized with a custom ASIC developed within the GADMC, which already provided very promising results in terms of signal-to-noise ratio and dynamic range at $77 \mathrm{~K}$. With 9 $\mathrm{V}$ of over voltage applied to the tile, the measured mean peak amplitude of the single photoelectron is $9.7 \mathrm{mV}$, translating to a linear dynamic range of $70 \mathrm{PE}$.

\section{References}

[1] P. A. Amaudruz et al., [DEAP-3600 Collaboration], "First Results from the DEAP-3600 Dark Matter Search with Argon at SNOLAB", Phys. Rev. Lett. 121, 071801, (2018)

[2] R. Ajaj et al., [DEAP-3600 Collaboration], "Search for dark matter with a 231-day exposure of liquid argon using DEAP-3600 at SNOLAB", Phys. Rev. D 100, 022004, (2019)

[3] P. Agnes et al., [DarkSide Collaboration], "First results from the DarkSide-50 dark matter experiment at Laboratori Nazionali del Gran Sasso", Phys. Lett. B 743, 456, (2015)

[4] P. Agnes et al., [DarkSide Collaboration], "Results from the first use of low radioactivity argon in a dark matter search", Phys. Rev. D 93, 081101, (2016)

[5] E. Aprile et al., [Xenon Collaboration], "Projected WIMP sensitivity of the XENONnT dark matter experiment", JCAP11 031, (2020) 
[6] D. Akerib et al., [LUX Collaboration], "Projected WIMP sensitivity of the LUX-ZEPLIN dark matter experiment", Phys. Rev. D 101, 351, (2020)

[7] P. Agnes et al., [DarkSide Collaboration], "DarkSide-50 532-day dark matter search with low-radioactivity argon", Phys. Rev. D 98, 102006, (2018)

[8] P. Agnes et al., [DarkSide Collaboration], "Low-Mass Dark Matter Search with the DarkSide50 Experiment", Phys. Rev. Lett. 121, 081307, (2018)

[9] E. Bagnaschi et al., "Likelihood analysis of the pMSSM11 in light of LHC 13-TeV data", Eur. Phys. J. C 78, 8738 (2018)

[10] P. Agnes et al., [DarkSide Collaboration], "Constraints on Sub-GeV Dark-Matter-Electron Scattering from the DarkSide-50 Experiment", Phys. Rev. Lett. 121, 111303, (2018)

[11] B. Abi et al. "The single-phase ProtoDUNE technical design report." arXiv preprint, arXiv:1706.07081 (2017).

[12] P. Agnes et al., [DarkSide Collaboration]," Separating ${ }^{39} \mathrm{Ar}$ from ${ }^{40} \mathrm{Ar}$ by cryogenic distillation with Aria for dark-matter searches", Eur. Phys. J. C 81, 359, (2021) 